\title{
Simulated electrical response of randomly distributed and aligned graphene/ polymer nanocomposites
}

DOI:

10.1016/j.compstruct.2018.03.022

\section{Document Version}

Accepted author manuscript

Link to publication record in Manchester Research Explorer

\section{Citation for published version (APA):}

Manta, A., Gresil, M., \& Soutis, C. (2018). Simulated electrical response of randomly distributed and aligned graphene/ polymer nanocomposites. Composite Structures. https://doi.org/10.1016/j.compstruct.2018.03.022

\section{Published in:}

Composite Structures

\section{Citing this paper}

Please note that where the full-text provided on Manchester Research Explorer is the Author Accepted Manuscript or Proof version this may differ from the final Published version. If citing, it is advised that you check and use the publisher's definitive version.

\section{General rights}

Copyright and moral rights for the publications made accessible in the Research Explorer are retained by the authors and/or other copyright owners and it is a condition of accessing publications that users recognise and abide by the legal requirements associated with these rights.

\section{Takedown policy}

If you believe that this document breaches copyright please refer to the University of Manchester's Takedown Procedures [http://man.ac.uk/04Y6Bo] or contact uml.scholarlycommunications@manchester.ac.uk providing relevant details, so we can investigate your claim.

\section{OPEN ACCESS}




\section{Accepted Manuscript}

Simulated electrical response of randomly distributed and aligned graphene/ polymer nanocomposites

Asimina Manta, Matthieu Gresil, Constantinos Soutis

PII:

S0263-8223(17)34256-3

DOI: https://doi.org/10.1016/j.compstruct.2018.03.022

Reference: COST 9469

To appear in:

\section{Composite Structures}

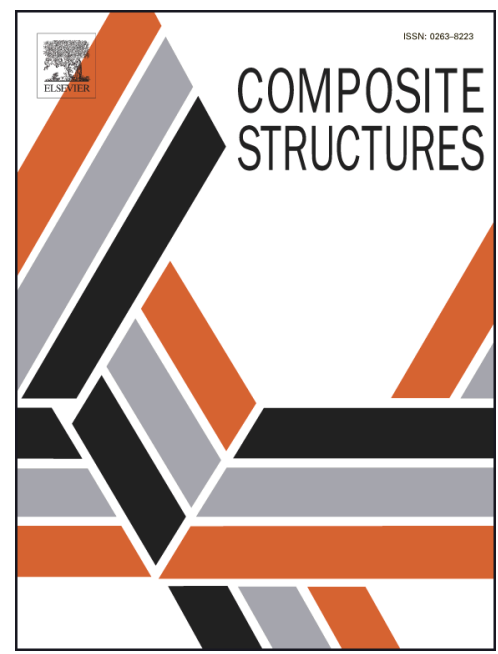

Received Date: $\quad 18$ December 2017

Revised Date: $\quad 27$ February 2018

Accepted Date: $\quad 8$ March 2018

Please cite this article as: Manta, A., Gresil, M., Soutis, C., Simulated electrical response of randomly distributed and aligned graphene/polymer nanocomposites, Composite Structures (2018), doi: https://doi.org/10.1016/ j.compstruct.2018.03.022

This is a PDF file of an unedited manuscript that has been accepted for publication. As a service to our customers we are providing this early version of the manuscript. The manuscript will undergo copyediting, typesetting, and review of the resulting proof before it is published in its final form. Please note that during the production process errors may be discovered which could affect the content, and all legal disclaimers that apply to the journal pertain. 


\title{
Simulated electrical response of randomly distributed and aligned graphene/polymer nanocomposites
}

\author{
Asimina Manta ${ }^{\mathrm{a}}$, Matthieu Gresil ${ }^{\mathrm{a}, \mathrm{b}}$, and ${ }^{*}$ Constantinos Soutis ${ }^{\mathrm{b}}$ \\ ${ }^{a}$ i-composites lab, School of Materials, University of Manchester, James Lighthill Building, \\ Sackville Street, Manchester M13 9PL, United Kingdom. \\ e-mail: asimina.manta@postgrad.manchester.ac.uk \\ e-mail: matthieu.gresil@manchester.ac.uk \\ ${ }^{\mathrm{b}}$ Aerospace Research Institute, Faculty of Engineering and Physical Sciences, University of \\ Manchester, James Lighthill Building, Sackville Street, Manchester M13 9PL, United \\ Kingdom. \\ e-mail: constantinos.soutis@manchester.ac.uk
}

\begin{abstract}
In this work, a recently developed numerical method that simulates the electrical response of a graphene/polymer nanocomposite is validated with experimental data. The approach is based on the multiscale method and consists of a unit cell and a representative volume element (RVE), accounting for aligned and randomly distributed nanoparticles. At the unit cell level, the material nano characteristics (filler geometry, constituent electrical and interfacial properties) are integrated into a local resistance algebraic matrix. The material architecture is then modelled at the micro-level (RVE) by a user-defined distribution of the unit cell electrical properties. A statistical sample was studied and the average electrical response was compared with measurements for direct $(D C)$ and alternate current $(A C)$. The proposed methodology accurately describes the nanocomposite electrical behaviour with its volume fraction and loading frequency. The model is proven to be an effective, flexible and timeefficient tool to design and optimize advanced nanocomposite systems.
\end{abstract}

Keywords: Nano-structures, Polymer, Graphene, Percolation Threshold, Finite Element Analysis (FEA), Computational modelling, Electrical Properties, Electrical Conductivity

\section{Introduction}

\subsection{Background}

Conductive polymers are considered for their potential applications in light emitting devices, batteries, electromagnetic shielding and piezoresistive sensors. In literature, there are several material systems proposed, including all the possible combinations of insulating polymer and conductive nano-micro-particle, such as carbon black [1-2], metallic powder [3-5], polyaniline [6], MWCNTs and graphite [7]. Great attention has also been paid to polymers reinforced by graphene and its derivatives, due to progress in their manufacturing process and attractive thermal and electrical behaviour [8-15]. ]. The effect of the multilayer graphene thickness and temperature on electrical conductivity was studied in [8], while the impact of synthesis on electrical performance was explored by studying compacts of graphene [9], graphene oxides produced by different synthesis routes [10] and reduced graphene oxides [11]. In [12], reinforcing polymer with graphene resulted in significant improvement of the thermal performance, while the thermal transport was studied for free-standing graphene nanoribbon as a function of temperature [13], and for suspended graphene sheets with different sizes [14]. Large-area reduced graphene oxide thin film was fabricated and examined by the authors in [15] that showed improved electrical and thermal conductivity with enhanced electromagnetic 
interference shielding compared to conventional small-area graphene oxide. The aforementioned studies demonstrated the high sensitivity of graphene electrical and thermal performance on the synthesis process and the ability they offer to customise material response. In addition, graphene sheets or graphene nanoplatelets (GNP) have been proven to form stable conductive networks [16] in a lower volume content due to their 2D shape and relatively high aspect ratio (AR). It should be noted that there is a large variation in the measured electrical behaviour [16-23], in terms of percolation threshold and electrical conductivity. Alkylfunctionalised graphene/PDMS was prepared exhibiting a percolation threshold of $0.63 \mathrm{vol} \%$ [16]. Expanded graphite (EG)/PMMA showed a percolation threshold of $0.6 \mathrm{vol} \%$ when prepared by direct solution blending of PMMA with EG [17], in the work of [18] it was found $1 \mathrm{wt} \%$, while for the graphite nanosheets/PMMA was set as low as 0.31vol\% [19]. In [20], graphene/polyurethane was manufactured with three different dispersion methods, where solvent mixed samples exhibited a percolation threshold of $0.3 \mathrm{vol} \%$, melt-intercalation samples showed $0.5 \mathrm{vol} \%$ and for in-situ polymerised composite samples the threshold was between 0.3 and $0.5 \mathrm{vol} \%$. The thermal, electrical and mechanical properties of silicone rubber reinforced with graphene nanoplatelets was studied, revealing a notable enhancement, although the percolation transition was not achieved even for $8 \mathrm{wt} \%$ loading [21]. In [22], graphene nanosheet/aluminum nitride composites were prepared by hot-pressing, showing typical percolation behaviour with a percolation threshold of $2.5 \mathrm{vol} \%$. Finally, hybrid graphene-carbon nanotube/polypropylene nanocomposites were manufactured to investigate the synergistic effect between the graphene and carbon nanotube particles on the composite's electrical performance [23]. In summary, the filler geometry, constituent properties and manufacturing processes in conjunction with the probabilistic nature of conduction, affect significantly the electrical response of the nanocomposite. This is giving the research engineer the opportunity to form extremely customised material structures corresponding to their design needs and means of production. However, investigating all these variables experimentally (by trial and error) becomes a time consuming, cost expensive and cumbersome exercise, especially when the probabilistic nature of the nanocomposite needs to be considered. As a consequence, a reliable numerical model is needed that can be used as an effective tool for the design optimisation and accurate prediction of the nanocomposite electrical performance.

\subsection{Electrical Simulation Models}

Although material preparation and experimental work exhibit high financial and time cost, there are only a few computational models that can accurately estimate the electrical response of graphene reinforced polymers. Many of them are poorly or not validated with experimental data. In literature, the electrical simulation models are divided into two main categories- the percolation threshold models and the ones predicting the electrical conductivity with nanocomposite's volume fraction and loading frequency. The percolation models proposed were taking into account the filler shape, aspect ratio, tunnelling distance, overlapping, and the formation of agglomerations. Oskouyi et al. [24] apply the Monte Carlo method to model the percolation threshold for disk-shaped fillers, while, Ambrosetti et al. [25] conducted a numerical study to investigate a system's percolative properties consisting of hard oblate ellipsoids of revolution surrounded with soft penetrable shells. Otten et al. [26] developed an analytical approach to investigate the percolation behaviour of polydisperse nanofillers of platelet-based composites but the effect of the filler properties distribution was not considered. Also, their modelling approach was subjected to certain limitations, like the platelet thickness and tunnelling decay length being of the same order of magnitude, or the disk-like filler diameter to be much larger than the filler thickness. Regarding the effect of reinforcing particles overlapping and agglomerations, Xia et al. [27] proposed a numerical simulation which is able to predict the percolation threshold for identical ellipses with the overlapping effect for a 2D structure, while Vovchenko et al. [28] predicted the percolation threshold of composites filled with intersecting circular discs in a 3D structure. Apart from the conventional approaches to predict the percolation threshold for materials reinforced with 2D particles, Mathew et al. 
[29] conducted a Monte Carlo study on the percolation of hard platelets in a 3D continuum system considering the rate of order in the microstructure, with the employment of isotropicnematic (IN) transition.

Despite the satisfactory number of methods predicting the percolation threshold of graphene/polymer nanocomposites, there are only a few studies simulating their electrical conductivity. Hicks et al. [30] developed a tunnelling-percolation model to investigate electrical transport in nanocomposites reinforced by rectangular 2D particles forming 2D networks. However, the application of this model is limited to the simulation of aligned graphene nanocomposites, while in common graphene reinforced nanocomposites, fillers exhibit a wide range of shapes and the conductive network formed is considered to be a $3 \mathrm{D}$ one. In a later work, Ambrosetti et al. [31] studied the electrical conductivity of an insulating matrix reinforced with conductive ellipsoids by assuming that an expected curve of electrical conductivity variation would be applied and finally be reduced in a geometrical form taking into account the inter-particle distance and the tunnelling distance. Oskouyi et al. [32] developed a 3D Monte Carlo model to study the percolation, conductivity, and piezoresistive behaviour of composites filled with randomly dispersed impenetrable conductive nano-disks. Finally, a continuum model to determine the effective AC and DC electrical properties of graphene nanocomposite was developed by Hashemi and Weng [33]. Their approach consisted of three major components, embodying the most fundamental characteristics of the graphene nanocomposites, i.e. percolation threshold, interface effects and the additional contribution of electron hopping and micro capacitor structures to interfacial properties. Considering all these numerical models, except of [33], the rest [30-32], did not provide sufficient proof of validation with relevant experimental studies. Although these results seem to be in accordance with physical observation, a straightforward point to point comparison with experimental data is missing, questioning the model's validity and applicability to different structures of nanocomposites.

In this paper, a multi-scale multi-physics finite element model is developed to describe the electrical response of recently tested graphene/polymer nanocomposites. It focuses on more realistic material systems accounting for irregularities and filler dispersion. To build the FE model, the nanocomposite architecture was identified in detail by defining the material phases and their electrical properties, particle average geometry (diameter and thickness) and filler distribution. Once, the properties were obtained, they were used at the unit cell level to model the local electrical conduction. Then, at the RVE scale, the already obtained electrical response was distributed in accordance with the decided particle distribution. A statistical sample was processed and the basic statistical features (average, standard deviation) were calculated and compared with experimental data. In terms of structure, different types of internal nanocomposite architecture were studied, such as the case of polymer intercalation among graphene sheets of graphene nanoplatelets, aligned graphene sheets through AC electric fields, random filler distribution and aligned graphene sheets through the hot-press manufacturing process.

\section{Methodology and Results}

\subsection{Model Overview}

To simulate the electrical response of experimental data published for certain nanocomposite material systems, the multi-scale multi-physics finite element model (FEM) presented in [34] and [35] is employed. The modelling approach consists of the creation of a unit cell and a micro-scale nanocomposite model (Representative Volume Element - RVE) on a commercially available finite element package (Multi-physics ANSYS APDL).

The unit cell consists of one graphene nanoplatelet surrounded by a thin layer of polymer in accordance with the hard-core penetrable shell approach $[25,29]$. This polymer layer 
represents the inter-plate volume between successive graphene reinforcements, in which the quantum conduction phenomena (tunnelling effect, electron hopping) take place. The main parameters derived from this model are the geometrical description of the nanoparticle (shape, dimensions), the electrical response of the nanoparticle in respect of its manufacturing process and the height of barrier being related to energy barrier between the nanoparticle and the insulator to be penetrated by the electrons [36]. The unit cell is loaded with a constant electric potential for the case of direct current (DC) response, or with an alternating current for the case of AC loading, so as to compute the resistance matrix representing this system in respect to the unit cell parameters and the loading frequency when AC loading is applied.

The RVE is a rectangular block, where its resistance matrix elements, obtained earlier through the unit cell, are distributed under a user-defined distribution. The block side is assumed to be 5 times the maximum nanoparticle dimension $d$ [34]. The unit cell material properties distribution represents the position of graphene in the bulk volume of the nanocomposite and it is influenced by the nanocomposite manufacturing process. Finally, the orientation of graphene is simulated by a 3D random orientation of the corresponding element local coordinate system. The range of orientation angle is affected by the manufacturing process and the application of electric or magnetic field could have introduced particle alignment, enabling the formation of conductive paths.

\subsection{Electrical Response under DC Loading}

In order to prove the model's ability to predict the electrical response of material systems of different structure and manufacturing process, two different sets of experimental data were used. The first one is the result of the experimental work performed by Chen et al. [19] related to nanocomposites prepared by in situ polymerisation of PMMA/graphite nanosheet (NanoG) composite films, while the second one is about aligned graphene flakes epoxy nanocomposite produced by Wu et al. [37].

\subsubsection{Model Validation by Chen et al. data [19]}

In this experimental work [19], expanded graphite was prepared according to the literature reports $[38,39]$, and the graphite nanosheets were made by immersing the expanded graphite in an aqueous alcohol solution and subjecting to powdering in an ultrasonic bath. The resulting graphite sheets had a diameter of 0.5-20 $\mu \mathrm{m}$ and thickness of 30-80 nm. In accordance with the stated manufacturing process, the graphite sheets are estimated to have an in-plane electrical conductivity of $6.94 \mathrm{~S} / \mathrm{m}$ [40]. Although the manufacturing process is not one-by-one identical to the one published, it could be an acceptable assumption since more reliable data were not available. Moreover, the inner structure of the graphite nanosheets was examined by TEM technique, showing that some of the nanosheets were composed of thinner sheets with a thickness of about 2-5 nm.

The nanocomposite material was fabricated by an in-situ polymerization method, where MMA (methyl methacrylate) monomer and NanoG were put in an ultrasonic bath, followed by the addition of BPO (benzoyl peroxide) and the sonication was kept on until the polymerisation took place and the suspension turned to solid. The conducting films of 50-100 $\mu \mathrm{m}$ were made by casting the dispersion on glass slides. This manufacturing process, when aided by the sonication of the graphite nanosheets with MMA monomers, could lead MMA monomers to penetrate into the nanospace inside the $30-80 \mathrm{~nm}$ thick NanoG resulting in thinner graphite sheets. The whole process and the resulted nanocomposite structure is summarised in Fig. 1.

In this numerical analysis, the graphite sheets were modelled as circular sheets with a diameter of $20 \mu \mathrm{m}$ and thickness of $2 \mathrm{~nm}$, while the height of barrier of the PMMA is estimated to be $1 \mathrm{eV}$ [41]. Each graphite sheet of thickness $2 \mathrm{~nm}$ is a part of a stack of graphite sheets with a thickness of $30 \mathrm{~nm}$, leading to a local distribution through-the-thickness of the graphite stack. This nanostructure feature could be approached by a macroscopic through-the specimen- 
thickness graphene distribution with a mean value $(\mu)$ equal to the half thickness of the RVE and standard deviation $\left(s_{d}\right)$ to be subject of parametric study.

In Fig. 2, the effect of standard deviation on the percolation probability and the percolation threshold (1) is studied, according to the work performed in [34]. The percolation probability $P\left(V_{f}\right)$ is the probability of a specimen to be conductive, and it is defined as the ratio of the number of conductive specimens to the total number of specimens examined for a stated volume fraction $\left(V_{f}\right)$. The percolation threshold $\left(V_{p}\right)$ is defined as the critical $V_{f}$ above that a nanocomposite is considered conductive. In statistical terms, the percolation threshold is found when the $50 \%$ of the statistical sample is conductive. The percolation probability for a given volume fraction rises with decreasing standard deviation $s_{d}$ of the filler distribution, while for $s_{d}=2.5 \mu \mathrm{m}$ the obtained percolation threshold is $V_{p}=0.58 \%$ comparable to the percolation threshold shown on the experimental curve (Fig. 2) being close to $0.55 \mathrm{vol} \%$.

$$
P\left(V_{f}\right)=\frac{1}{2}\left(1+\tanh \left(A\left(V_{f}-V_{p}\right)\right)\right)
$$

As far as the variation of electrical conductivity as a function of the $V_{f}$ is concerned, in Fig. 3 the comparison between the experimental curve and the computational curves is presented. It is shown that for standard deviation $s_{d}=2.5 \mu \mathrm{m}$ the experimental results were better represented by the simulation curve lying among the experimental points. It could be concluded that the distribution of the filler is not common for volume fraction, since the volume fraction changes significantly the homogeneity of the material systems. Increasing $V_{f}$ arises two main phenomena in the nanocomposite structure with opposing effects on the electrical conductivity - the formation of conductive paths and the formation of agglomerations. For $V_{f}$ around the percolation threshold, the increase of conductive paths has a greater influence on conductance compared to the limited formation of agglomerations. For $V_{f}$ ranging between $2.5 \%$ and $4 \%$, the increasing agglomerates are reducing the conductive paths, thus requesting higher $s_{d}$ on the filler distribution. Finally, for $V_{f}>4 \%$ the two phenomena are shown to be balanced and agglomerates to have less effect. To predict these phenomena and bound the resulting electrical response, the same analysis could be run for two extreme values of standard deviation, lower and higher than $s_{d}=2.5 \mu \mathrm{m}$.

\subsubsection{Model Validation by Wu et al. data [37]}

In this experimental work performed by Wu et al.[37], graphene nanoplatelets (GnPs) with a diameter of $25 \mu \mathrm{m}$ and an average thickness of $6 \mathrm{~nm}$ were commercially obtained (XG Science). The suggested epoxy nanocomposites with contents of $0.27,0.54 .0 .81$ and $1.08 \mathrm{vol} \%$ were fabricated by combining sonication and calendaring processes. At first, the GnPs were mixed with the liquid epoxy resin using ultrasonic homogenizer, and the mixture was then further processed by a three-roll mill. After this dispersion process, the hardener was added. Finally, the GnPs were aligned by using an AC electric field.

In this case, at first the nanocomposite with random filler orientation was modelled for comparison and then the architecture of the nanocomposite with aligned graphene particles was approached. Regarding the aligned $\mathrm{GnP}$ nanocomposite, the architecture of the experimental material was studied and these conclusions were applied to our modelling approach. In Figure $1 \mathrm{~d}$ of ref [37], the optical micrograph of GnPs in the liquid epoxy resin for $0.054 \mathrm{vol} \%$ after the application of the AC electric field for 20min is displayed. It could be seen that discrete paths of GnPs were created along the specimen, portrayed in reconstructed figure Fig. 4. This finding, based on the optical observation, is further supported by the results by appropriate image processing of the micrograph calculating the variation of $\mathrm{GnP}$ concentration transverse to the GnP paths. In Fig. 4, it could be calculated that by setting a concentration threshold of 10\%, 212 peaks were found, on a specimen with a width of $1075.758 \mu \mathrm{m}$, resulting on average to 0.197 GnP paths per $1 \mu \mathrm{m}$ width. Therefore, an additional distribution is applied to simulate the aligned structure of the GnPs. The number of elements transverse to the alignment direction is 
multiplied by the number of $\mathrm{GnP}$ paths/ $\mu \mathrm{m}$ obtained previously and the resulting number is the number of paths to be created longitudinal to the specimen. In accordance to this, it is expected to have 6 paths and a parametrical study for 4, 5, 6 and 10 paths is performed to investigate the effect of $\mathrm{GnP}$ paths density on the electrical performance of the nanocomposite.

The effect of the GnP paths density on the percolation probability is depicted in Fig. 5. Decreasing the GnP paths density, the obtained percolation threshold decreased significantly, as expected. The GnP particles are aggregated to specific areas longitudinal to the specimen length forming specific discrete paths. Decreasing GnP paths density leads to less discrete paths which are, nevertheless, complete and more intense to cover the specimen full length and carry the electrical load.

From Fig. 6, it could be noted that there is not a specific value for the GnP paths density able to simulate the nanocomposite structure for every volume fraction, while this result could be supported by physical/experimental observations. Increasing volume fraction leads to increasing number of particles being able to form paths, therefore, leading to the creation of more conductive paths. In addition to this, increase in $\mathrm{GnP}$ content could lead to decreased $\mathrm{GnP}$ mobility, creating locally paths which would raise the average path density. For a specific value of $V_{f}$, the increase in GnP path density reduces the electrical conductivity. For every value of $V_{f}$ a specific number of particles is available, and when GnP path density is increased this constant amount of particle is distributed to many paths, resulting to particle paths with a poor connection (bigger gaps as particles are further apart).

\subsection{Electrical Response under AC Loading}

The ability of the model to predict the electrical response of material systems under alternating current was explored and two different sets of experimental data were used. The first one is the result of the experimental work performed by Yu et al. [42] related to nanocomposites prepared by a combination of in situ polymerisation and solution method of PVDF/reduced graphene sheets composite, while the second one is about the experimental work by Shang et al. [43], where oriented PVDF/graphene nanosheets nanocomposite was fabricated by compression - moulding.

\subsubsection{Model Validation by Yu et al. data [42]}

In this experimental work [42], reduced graphene sheets were fabricated by heating GOs, obtained from natural graphite with the modified Hummers method. The graphene sheets in aqueous solution exhibited an average thickness of $1 \mathrm{~nm}$, while the average diameter was considered around $2 \mu \mathrm{m}$ with a minimum value of $0.1 \mu \mathrm{m}$ and a maximum value of $7 \mu \mathrm{m}$ [42]. The nanocomposite material was prepared by a solution method and the dried mixture was compressed into films with a thickness of around $420 \mu \mathrm{m}$ at $180^{\circ} \mathrm{C}$ under a pressure of about $10 \mathrm{MPa}$

The nanocomposite architecture was approached by a random distribution of the graphene sheets in the polymeric volume. Two graphene sheets cases were considered - the first one has a diameter of $2 \mu \mathrm{m}$ and the second one has a diameter of $0.1 \mu \mathrm{m}$, both with a thickness of $1 \mathrm{~nm}$. The graphene sheets were assumed to have an in-plane conductivity of $350 \mathrm{~S} / \mathrm{m}$ and relative permittivity of 15 . In addition to this, in the unit cell scale, the Maxwell - Wagner Sillars interfacial polarization [44] was taken into account. The resulting $\varepsilon_{\text {net }}^{*}$ was used to model the dielectric permittivity of the polymer layer surrounding the graphene sheets $(\mathbf{2})$, where $\varepsilon_{\text {cond }}^{*}$ is the complex relative permittivity of the conductive component, $\varepsilon_{i n s}^{*}$ is the complex relative permittivity of the insulator and $\mathrm{x}$ is the insulator volume fraction. The created model was solved for a range of frequencies $10^{2}-10^{6} \mathrm{~Hz}$. The complex relative permittivity is defined as (3) with the real part being the relative permittivity $\varepsilon\left(\varepsilon_{r}=\varepsilon\right)$ and the imaginary part being $\varepsilon_{i}=\frac{\sigma}{2 \pi f \varepsilon_{o}}$ ( $\sigma$ is electrical conductivity, $f$ is the frequency and $\varepsilon_{o}$ is the permittivity of free space), combining both the relative permittivity and the electrical conductivity of the material. 


$$
\begin{aligned}
& \varepsilon_{\text {net }}^{*}=\frac{\varepsilon_{\text {ins }}^{*} \cdot \varepsilon_{\text {cond }}^{*}}{x \cdot \varepsilon_{\text {cond }}^{*}+(1-x) \cdot \varepsilon_{\text {ins }}^{*}} \\
& \varepsilon^{*}=\varepsilon_{r}-j \varepsilon_{i}
\end{aligned}
$$

The effect of the simulation parameters on the analysis results compared to the experimental data for frequency $f=100 \mathrm{~Hz}$, is shown in Fig. 7 and Fig. 8. It could be concluded that the best correlation is achieved by the combination of $x=10^{-12}$, graphene in-plane conductivity $\sigma=350 \mathrm{~S} / \mathrm{m}$ and graphene diameter $0.1 \mu \mathrm{m}$. According to [11], the GO films, obtained from the petri dish after drying, exhibit low electrical conductivity and they should be treated with hydrohalic acids as reducing agents to recover the electrical conductivity to possible extent. Moreover, $\mathrm{GO}$ is not stable in high temperature as holes are formed by $\mathrm{CO}_{2}$ elimination, thus reducing its electrical conductivity. Comparing the procedure of [11] with the experimental procedure of [42], it could be concluded that the graphene sheets obtained in [42] have much lower electrical conductivity than the expected value $\left(\approx 2 \cdot 10^{3}-10^{5} \mathrm{~S} / \mathrm{m}\right)$, supported by the findings of the numerical analysis compared to the experimental measurements. Finally, regarding the filler size, the best correlation was achieved when the minimum graphene sheet diameter was considered. Due to the ultrasonic wave and the stirring applied, collisions among graphene particles may take place, breaking the particles and hence reducing their size.

Therefore, the distribution of the filler diameter when the filler is in an aqueous solution, is not really representative of the final product.

In Fig. 9 and Fig. 10, the variation of electrical conductivity and relative permittivity in respect of the alternate current frequency and the nanocomposite weight fraction is presented, considering in-plane filler electrical conductivity of $350 \mathrm{~S} / \mathrm{m}$ and Maxwell - Wagner - Sillars insulator volume fraction $x=10^{-12}$. The experimental electrical conductivity and relative permittivity are well correlated with the simulation results, giving confidence to assumptions and simplifications made.

\subsubsection{Model Validation with Shang et al. [43]}

Shang et al. [43] presented the fabrication and dielectric properties of oriented PVDF nanocomposites incorporated with graphene sheets. Although, the manufacturing process is the same in general with the one presented by the Yu et al. [42], there are some slightly different steps followed able to result in a different electrical response and architecture. The GO particles were reduced in the presence of PVDF particles obtaining GNS/PVDF solution, compared to the work [42], where the graphene sheets were fabricated by heating GO separately. Finally, the GNS/PVDF solution was drop cast in a glass plate and kept in an oven at $80^{\circ} \mathrm{C}$ for $3 \mathrm{~h}$ for the solvent to be slowly evaporated and obtain the GNS/PVDF composite films, while the final pieces were compression-moulded into steel board at $210^{\circ} \mathrm{C}$ for $20 \mathrm{~min}$ at a pressure of $15 \mathrm{MPa}$. It should be noted that the compression moulding conditions were higher than the ones in [42], contributing to the different material structure obtained, as it was observed that the graphene sheets exhibited some degree of alignment. In addition to this, in accordance with the results obtained in work [35], it could be assumed that the in-plane filler distribution could be random due to the solution casting of the films, whereas a through-the-thickness Gaussian distribution could be assumed due to the hot-pressing of the films.

Taking into consideration the manufacturing process, it could be estimated that the electrical conductivity of graphene sheets is $7 \mathrm{~S} / \mathrm{m}$, since the GO sheets reduction took place in presence of the PVDF particles, the recovery of graphene sheets' electrical conductivity could not be complete. The filler diameter was considered to be $2 \mu \mathrm{m}$ and its thickness was set equal to $10 \mathrm{~nm}$ as it was stated in [43]. Moreover, the interphase developed between the graphene sheets and the PVDF matrix is expected to be different than the usually obtained. As a consequence, the height of barrier was estimated to be equal to $2.5 \mathrm{eV}$, while the insulator volume fraction found on the Maxwell - Wagner - Sillars equation was assumed $x=10^{-16}$, significantly smaller 
than the value estimated previously. Since the GO sheets were reduced in the presence of the PVDF particles, it is expected a more fine and thinner interphase to be produced.

In Fig. 11 and Fig. 12, the simulation results obtained for standard deviation $s_{d}=1.64 \mu \mathrm{m}$ of the through - the - thickness filler distribution are compared with the corresponding experimental ones [42]. A good correlation between numerical and measured data is achieved mainly at the lowest and highest volume fraction. Although for the rest of the volume fractions there is a discrepancy, overall the model could be considered as providing an acceptable description of this nanocomposite type response. Moreover, these volume fractions are close to the percolation threshold and considering that the statistical samples around this threshold exhibit significantly high standard deviation due to the variant percolation probability, the experimental data are expected to differ from the average value of the analysed numerical sample.

\section{Concluding Remarks}

The validation of the model presented in [34] and [35] examining the effect of the nanocomposite architecture based on a number of experimental case studies, has been performed. Four different experimental data sets corresponding to different excitations (DC and $\mathrm{AC}$ ) and different nanocomposite structures (random and aligned) obtained from some of the most common and up-to-date manufacturing processes have been studied. It could be seen that the proposed model was able to simulate successfully the electrical response of the nanocomposites studied, while by appropriately varying the material properties/filler distribution applied at the RVE scale, any nanocomposite architecture could be approached with great accuracy, without being necessary to create complicated geometries to analyse. In addition to this, although in most cases, filler characteristics (diameter and thickness) distribution was not stated, some approximation could be made by using a constant value around the average of the distribution. However, one of the most challenging parts of our modelling is the assumptions regarding the material properties corresponding to the filler and the interphase developed between the matrix and the filler. There is high uncertainty on the determination of the material properties, since the effect of the manufacturing process of the filler and the nanocomposite is not clearly reported in the literature. An inaccurate estimation of constituent properties used in the model could result in misleading conclusions, in terms of the value of the relative permittivity and hence electrical conductivity. The type of the function though is expected to be independent of the absolute values used.

Finally, once the modelling was adapted to the special characteristics of the nanocomposite examined, it could be easily noticed that the obtained numerical response of the materials system, was in accordance with the physical observations, while the experimental data were found to lie between the limits of the statistical sample. The higher the volume fraction was, the lower the statistical range would be, since increasing volume fraction corresponded to higher percolation probability and therefore to a more homogeneous sample of material structure for a given volume fraction. Taking everything into consideration, it could be concluded that the suggested modelling procedure is successful and able to simulate accurately the electrical response of a wide range of different architectures under the most common excitations - DC and AC. The numerical model could also be used to design and optimise new improved nanocomposite systems, reducing the need for experiments based on trial and error that can be an expensive exercise. 


\section{Acknowledgments}

The presented work was conducted as part of a 3-Year PhD Research Project at the School of Materials, University of Manchester and funded by the President's Doctoral Scholar (PDS) Award.

\section{References}

[1] Sichel EK, Gittleman JI, Sheng P. Electrical properties of carbon-polymer composites. J Electron Mater 1982;11:699-747. doi:10.1007/BF02672392.

[2] Ishigure $\mathrm{Y}$, Iijima S, Ito H, Ota T, Unuma H, Takahashi M, et al. Electrical and elástic properties of conductor-polymer composites. J Mater Sci 1999;34:2979-85. doi:10.1023/A:1004664225015.

[3] Pinto G, Jiménez-Martín A. Conducting aluminum-filled nylon 6 composites. Polym Compos 2001;22:65-70. doi:10.1002/pc.10517.

[4] Roldughin VI, Vysotskii V V. Percolation properties of metal-filled polymer films, structure and mechanisms of conductivity. Prog Org Coatings 2000;39:81-100. doi:10.1016/S0300-9440(00)00140-5.

[5] Flandin L, Bidan G, Brechet Y, Cavaillé JY. New nanocomposite materials made of an insulating matrix and conducting fillers: Processing and properties. Polym Compos 2000;21:165-74. doi:10.1002/pc.10174.

[6] Ray SS, Biswas M. Water-dispersible conducting nanocomposites of polyaniline and poly(N-vinylcarbazole) with nanodimensional zirconium dioxide. Synth Met 2000;108:231-6. doi:10.1016/S0379-6779(99)00258-1.

[7] Quivy A, Deltour R, Jansen AGM, Wyder P. Transport phenomena in polymer-graphite composite materials. Phys Rev B 1989;39:1026-30. doi:10.1103/PhysRevB.39.1026.

[8] Fang X-Y, Yu X-X, Zheng H-M, Jin H-B, Wang L, Cao M-S. Temperature- and thickness-dependent electrical conductivity of few-layer graphene and graphene nanosheets. vol. 379. 2015. doi:10.1016/j.physleta.2015.06.063.

[9] Marinho B, Ghislandi M, Tkalya E, Koning CE, De With G. Electrical conductivity of compacts of graphene, multi-wall carbon nanotubes, carbon black, and graphite powder. Powder Technol 2012;221:351-8. doi:10.1016/j.powtec.2012.01.024.

[10] Kim YJ, Kahng YH, Kim N, Lee J-H, Hwang Y-H, Lee M, et al. Impact of synthesis routes on the chemical, optical, and electrical properties of graphene oxides and its derivatives. Curr Appl Phys 2015;15:1435-44. doi:10.1016/j.cap.2015.08.008.

[11] Mohan VB, Brown R, Jayaraman K, Bhattacharyya D. Characterisation of reduced graphene oxide: Effects of reduction variables on electrical conductivity. Mater Sci Eng B 2015;193:49-60. doi:10.1016/j.mseb.2014.11.002.

[12] Gresil M, Wang Z, Poutrel Q-A, Soutis C. Thermal Diffusivity Mapping of Graphene Based Polymer Nanocomposites. Sci Rep 2017;7:5536. doi:10.1038/s41598-017-058660 .

[13] Xie H, Chen L, Yu W, Wang B. Temperature dependent thermal conductivity of a freestanding graphene nanoribbon. Appl Phys Lett 2013;102:111911. doi:10.1063/1.3136860.

[14] Chen L, Xie H, Yu W, Wang B, Wu Z. Thermal transport behaviors of suspended graphene sheets with different sizes. Int J Therm Sci 2015;94:221-7. doi:10.1016/j.ijthermalsci.2015.03.010. 
[15] Kumar P, Shahzad F, Yu S, Hong SM, Kim Y-H, Koo CM. Large-area reduced graphene oxide thin film with excellent thermal conductivity and electromagnetic interference shielding effectiveness. Carbon N Y 2015;94:494-500. doi:10.1016/j.carbon.2015.07.032.

[16] Hou Y, Wang D, Zhang X-M, Zhao H, Zha J-W, Dang Z-M. Positive piezoresistive behavior of electrically conductive alkyl-functionalized graphene/ polydimethylsilicone nanocomposites. J Mater Chem C 2013;1:515-21. doi:10.1039/c2tc00114d.

[17] Zheng W, Wong S-C, Sue H-J. Transport behavior of PMMA/expanded graphite nanocomposites. Polymer (Guildf) 2002;73:6767-73.

[18] Zheng W, Wong S-C. Electrical conductivity and dielectric properties of PMMA/expanded graphite composites. Compos Sci Technol 2003;63:225-35.

[19] Chen G, Weng W, Wu D, Wu C. PMMA/graphite nanosheets composite and its conducting properties. Eur Polym J 2003;39:2329-35. doi:10.1016/j.eurpolymj.2003.08.005.

[20] Kim H, Miura Y, Macosko CW. Graphene/Polyurethane Nanocomposites for Improved Gas Barrier and Electrical Conductivity. Chem Mater 2010;22:3441-50. doi: $10.1021 / \mathrm{cm} 100477 \mathrm{v}$.

[21] Song Y, Yu J, Yu L, Alam FE, Dai W, Li C, et al. Enhancing the thermal, electrical, and mechanical properties of silicone rubber by addition of graphene nanoplatelets. Mater Des 2015;88:950-7. doi:10.1016/j.matdes.2015.09.064.

[22] Yun C, Feng Y, Qiu T, Yang J, Li X, Yu L. Mechanical, electrical, and thermal properties of graphene nanosheet/aluminum nitride composites. Ceram Int 2015;41:8643-9. doi:10.1016/j.ceramint.2015.03.075.

[23] Al-Saleh MH. Electrical and mechanical properties of graphene/carbon nanotube hybrid nanocomposites. Synth Met 2015;209:41-6. doi:10.1016/j.synthmet.2015.06.023.

[24] Oskouyi AB, Mertiny P. Monte Carlo model for the study of percolation thresholds in composites filled with circular conductive nano-disks. Procedia Eng 2011;10:403-8. doi:10.1016/j.proeng.2011.04.068.

[25] Ambrosetti G, Johner N, Grimaldi C, Danani A, Ryser P. Percolative properties of hard oblate ellipsoids of revolution with a soft shell. Phys Rev E 2008;78:061126:1061126:11. doi:10.1103/PhysRevE.78.061126.

[26] Otten RHJ, Van Der Schoot P. Connectivity percolation of polydisperse anisotropic nanofillers. J Chem Phys 2011;134:094902:1-094902:15. doi:http://dx.doi.org/10.1063/1.3559004.

[27] Xia W, Thorpe MF. Percolation properties of random ellipses. Phys Rev A 1988;38:2650-6. doi:10.1103/PhysRevA.38.2650.

[28] Vovchenko L, Vovchenko V. Simulation of percolation threshold in composites filled with conducting particles of various morphologies. Materwiss Werksttech 2011;42:70-4. doi:10.1002/mawe.201100734.

[29] Mathew M, Schilling T, Oettel M. Connectivity percolation in suspensions of hard platelets. Phys Rev E 2012;85:61407. doi:10.1103/PhysRevE.85.061407.

[30] Hicks J, Behnam A, Ural A. A computational study of tunneling-percolation electrical transport in graphene-based nanocomposites. Appl Phys Lett 2009;95:213103:1213103:3. doi:http://dx.doi.org/10.1063/1.3267079.

[31] Ambrosetti G, Grimaldi C, Balberg I, Maeder T, Danani A, Ryser P. Solution of the 
tunneling-percolation problem in the nanocomposite regime. Phys Rev B 2010;81:155434. doi:10.1103/PhysRevB.81.155434.

[32] Oskouyi A, Sundararaj U, Mertiny P. Tunneling Conductivity and Piezoresistivity of Composites Containing Randomly Dispersed Conductive Nano-Platelets. Materials (Basel) 2014;7:2501-21. doi:10.3390/ma7042501.

[33] Hashemi R, Weng GJ. A theoretical treatment of graphene nanocomposites with percolation threshold, tunneling-assisted conductivity and microcapacitor effect in AC and DC electrical settings. Carbon N Y 2016;96:474-90. doi:10.1016/j.carbon.2015.09.103.

[34] Manta A, Gresil M, Soutis C. Multi-scale finite element analysis of graphene/polymer nanocomposites electrical performance. Proc. VII Eur. Congr. Comput. Methods Appl. Sci. Eng. (ECCOMAS Congr. 2016), vol. 1, Athens: Institute of Structural Analysis and Antiseismic Research School of Civil Engineering National Technical University of Athens (NTUA) Greece; 2016, p. 1984-2002. doi:10.7712/100016.1936.7586.

[35] Manta A, Gresil M, Soutis C. Predictive Model of Graphene Based Polymer Nanocomposites: Electrical Performance. Appl Compos Mater 2017;24:281-300. doi:10.1007/s10443-016-9557-5.

[36] Simmons JG. Generalized Formula for the Electric Tunnel Effect between Similar Electrodes Separated by a Thin Insulating Film. J Appl Phys 1963;34:1793-803. doi:10.1063/1.1702682.

[37] Wu S, Ladani RB, Zhang J, Bafekrpour E, Ghorbani K, Mouritz AP, et al. Aligning multilayer graphene flakes with an external electric field to improve multifunctional properties of epoxy nanocomposites. Carbon N Y 2015;94:607-18. doi:10.1016/j.carbon.2015.07.026.

[38] Chuan X, Chen D, Zhou X. Intercalation of $\mathrm{CuCl} 2$ into expanded graphite. Carbon N Y 1997;35:311-3. doi:10.1016/S0008-6223(97)85363-8.

[39] Kenji Fukuda, Kazuhiko Kikuya, Kennichi Isono, Masaki Yoshio. Foliated natural graphite as the anode material for rechargeable lithium-ion cells. J Power Sources 1997;69:165-8. doi:10.1016/S0378-7753(97)02568-8.

[40] Wang A, Chung DDL. Dielectric and electrical conduction behavior of carbon paste electrochemical electrodes, with decoupling of carbon, electrolyte and interface contributions. Carbon N Y 2014;72:135-51. doi:10.1016/j.carbon.2014.01.066.

[41] Mason TA, Johnson OK, Seegmiller DB, Fullwood DT, Dattelbaum AM, Mara NA, et al. A New Technique to Measure Tunneling Barrier Height in Solid Media. SAMPE 2011 Conf., 2011.

[42] Yu J, Huang X, Wu C, Jiang P. Permittivity, Thermal Conductivity and Thermal Stability of Poly(vinylidene fluoride)/Graphene Nanocomposites. IEEE Trans Dielectr Electr Insul 2011;18:478-84.

[43] Shang J, Zhang Y, Yu L, Shen B, Lv F, Chu PK. Fabrication and dielectric properties of oriented polyvinylidene fluoride nanocomposites incorporated with graphene nanosheets. Mater Chem Phys 2012;134:867-74. doi:10.1016/j.matchemphys.2012.03.082.

[44] Samet M, Levchenko V, Boiteux G, Seytre G, Kallel A, Serghei A. Electrode polarization vs. Maxwell-Wagner-Sillars interfacial polarization in dielectric spectra of materials: Characteristic frequencies and scaling laws. J Chem Phys 2015;142:194703. doi:10.1063/1.4919877. 
Fig. 1 Schematic representation of the in situ polymerisation and the nanocomposite structure based on [19]

Fig. 2 Percolation Probability vs $\mathrm{GnP}$ volume fraction $\left(\mathrm{V}_{\mathrm{f}}\right)$ for random and normal distributions of graphite nanosheets through-the-sample thickness.

Fig. 3 Electrical conductivity vs $\mathrm{V}_{\mathrm{f}}$, experimental data [19] are compared to numerical predictions (lower bound plotted for st. deviation $\mathrm{s}_{\mathrm{d}}=6.67 \mu \mathrm{m}$ and upper bound for $\mathrm{s}_{\mathrm{d}}=1.25 \mu \mathrm{m}$ )

Fig. 4 Image reconstruction of $\mathrm{Wu}$ et al. [37] figure 1d and processing for the calculation of $\mathrm{GnP}$ concentration transverse to the GnP paths.

Fig. 5 Percolation Probability variation as a function of volume fraction $\mathrm{V}_{\mathrm{f}}$ and $\mathrm{GnP}$ paths density

Fig. 6 Comparison between experimental data [37] and numerical results for different GnP paths density assuming randomly oriented $\mathrm{GnP}$

Fig. 7 Variation of electrical conductivity as a function of weight fraction at $100 \mathrm{~Hz}$ for different graphene sheets diameter $d$, graphene in-plane conductivity $\sigma$ and Maxwell - Wagner - Sillars insulator volume fraction $\mathrm{x}$

Fig. 8 Relative permittivity against weight fraction at $100 \mathrm{~Hz}$ considering different values of graphene sheets diameter d, graphene in-plane conductivity $\sigma$ and Maxwell - Wagner - Sillars insulator volume fraction $\mathrm{x}$

Fig. 9 Electrical conductivity vs weight fraction for frequencies of 102-106Hz (Graphene sheets $\mathrm{d}=0.1 \mu \mathrm{m}, \sigma=350 \mathrm{~S} / \mathrm{m}$ and $\mathrm{x}=10^{-12}$ )

Fig. 10 Relative permittivity vs weight fraction for frequencies of $10^{2}-10^{6} \mathrm{~Hz}$ (Graphene sheets $\mathrm{d}=0.1 \mu \mathrm{m}, \sigma=350 \mathrm{~S} / \mathrm{m}$ and $\mathrm{x}=10^{-12}$ )

Fig. 11 Electrical conductivity vs volume fraction for frequencies of $10^{2}-10^{6} \mathrm{~Hz}\left(\mathrm{~s}_{\mathrm{d}}=1.64 \mu \mathrm{m}\right.$, $\sigma=7 \mathrm{~S} / \mathrm{m}, \mathrm{x}=10^{-16}$ )

Fig. 12 Relative permittivity vs volume fraction for frequencies of $10^{2}-10^{6} \mathrm{~Hz}\left(\mathrm{~s}_{\mathrm{d}}=1.64 \mu \mathrm{m}\right.$, $\sigma=7 \mathrm{~S} / \mathrm{m}, \mathrm{x}=10^{-16}$ ) 


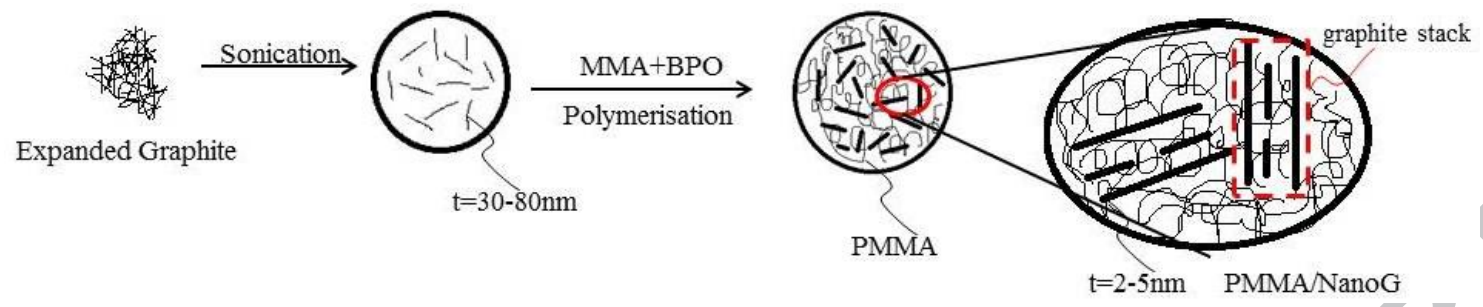




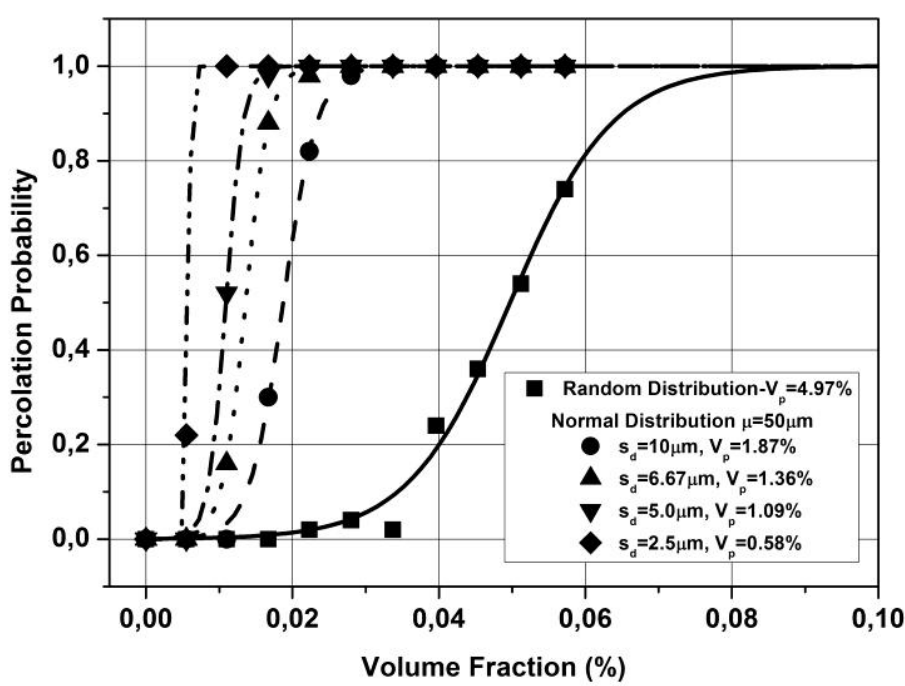




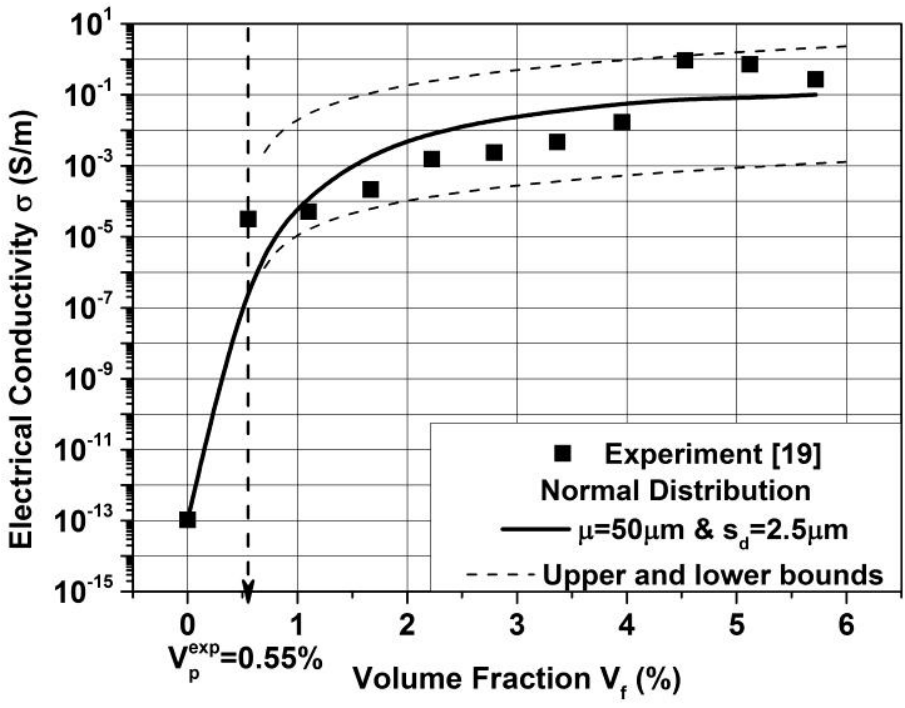




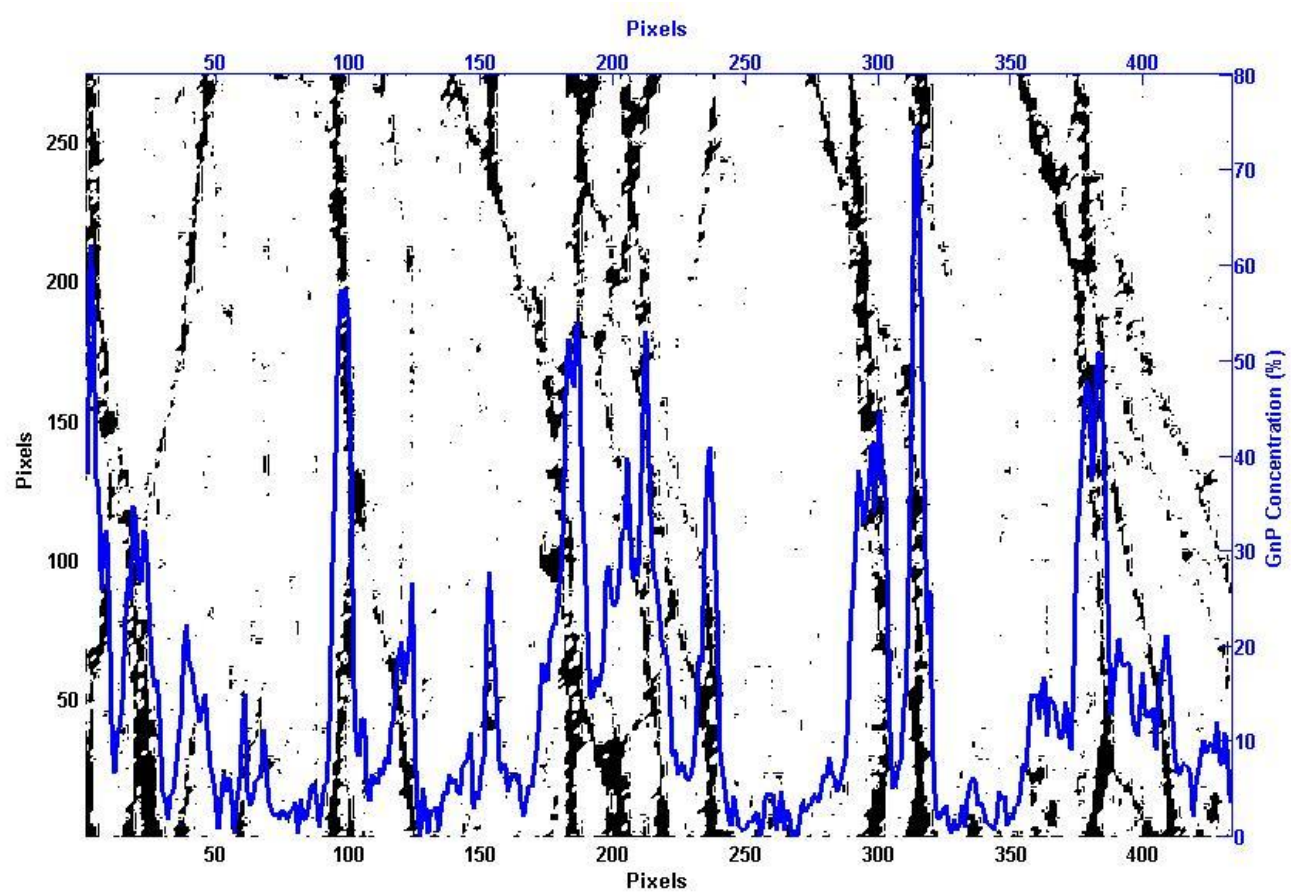




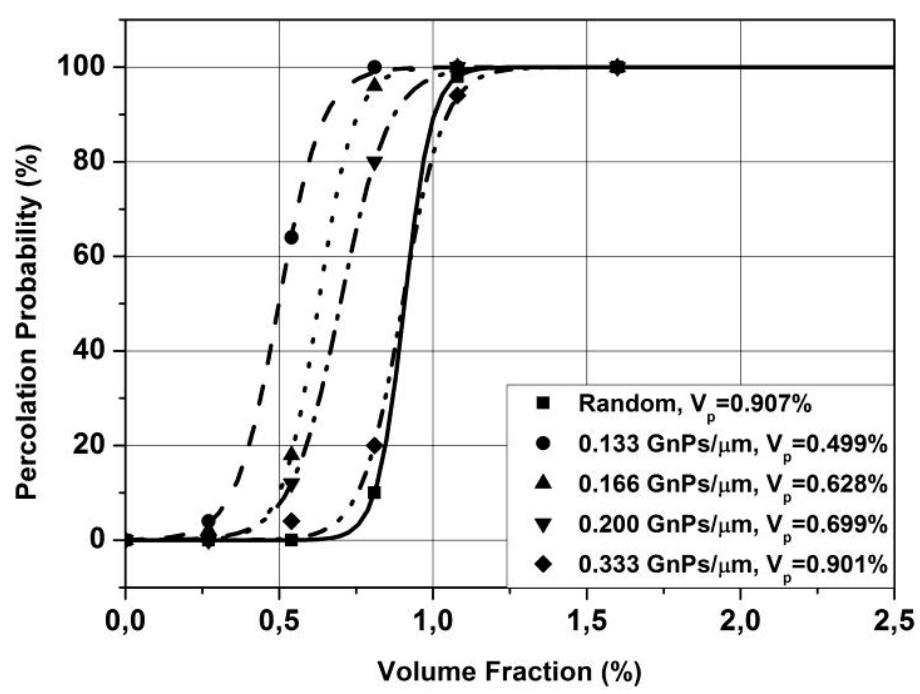




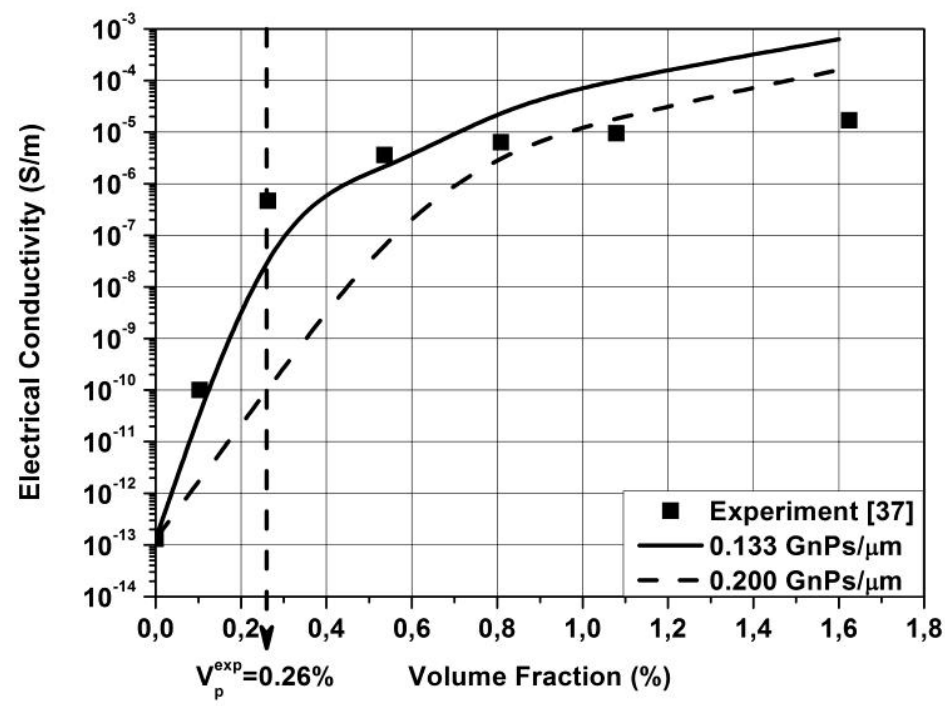




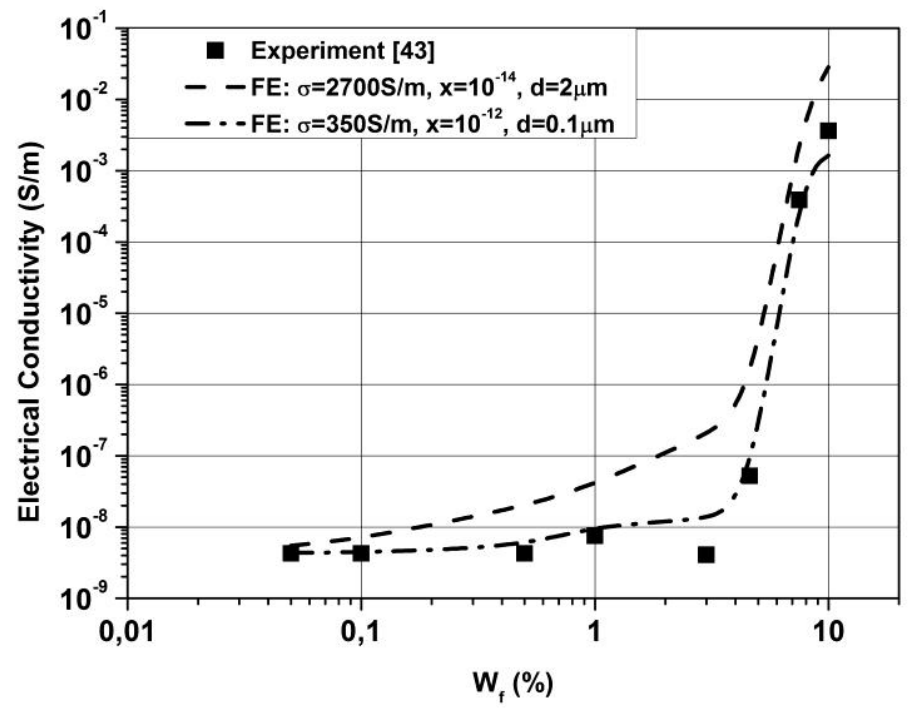




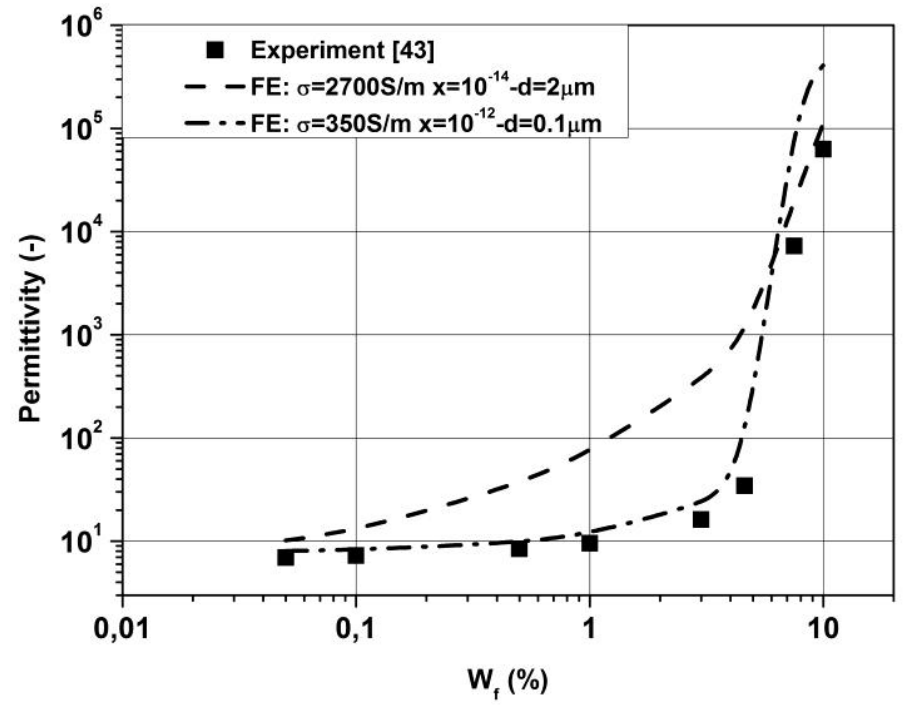



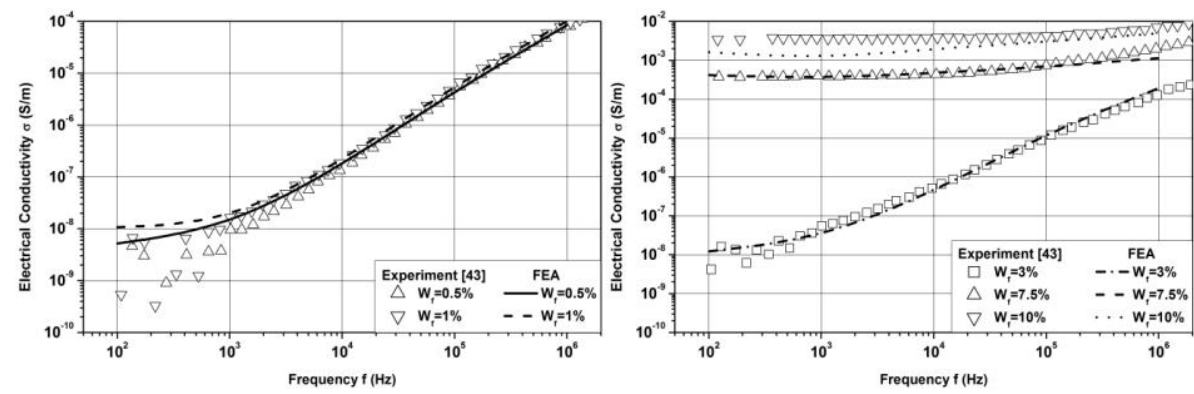

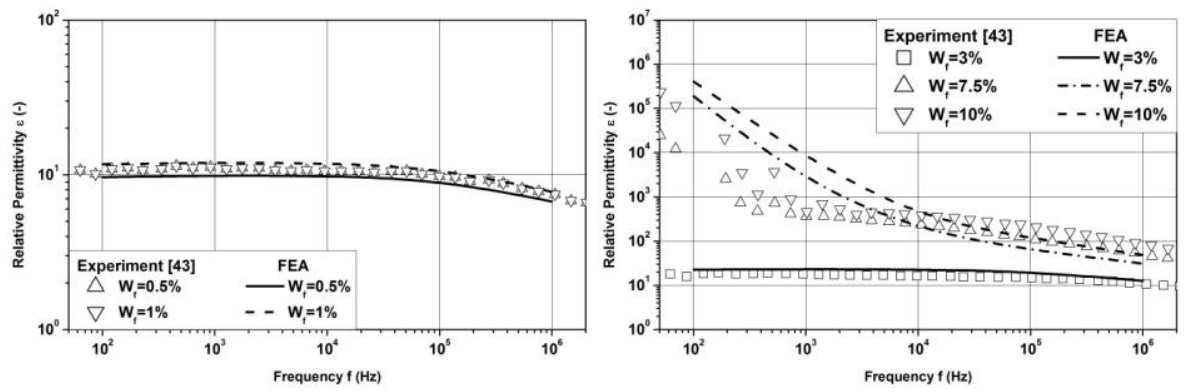


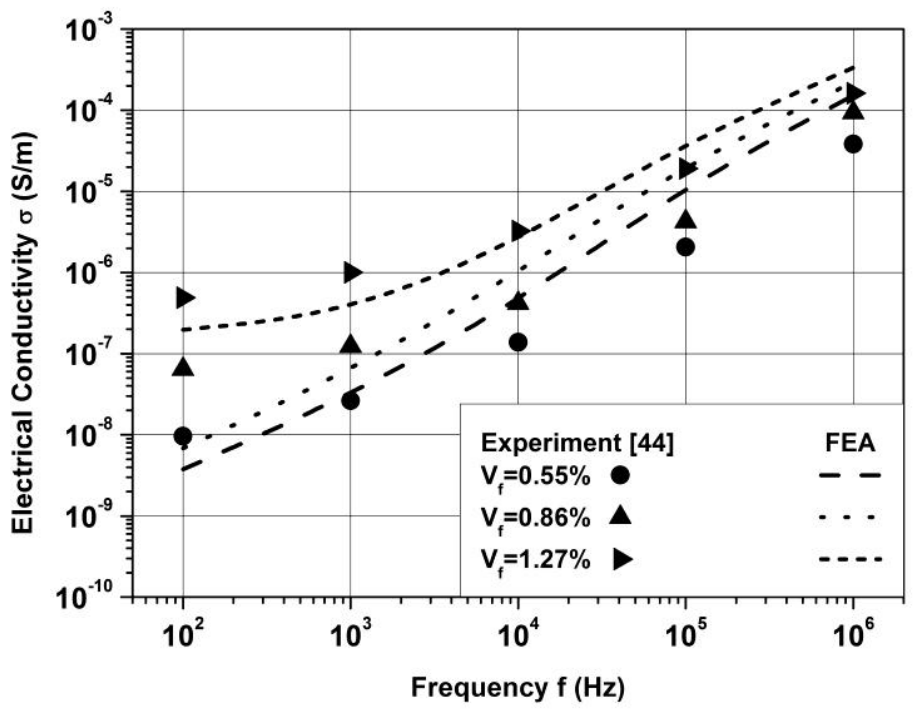




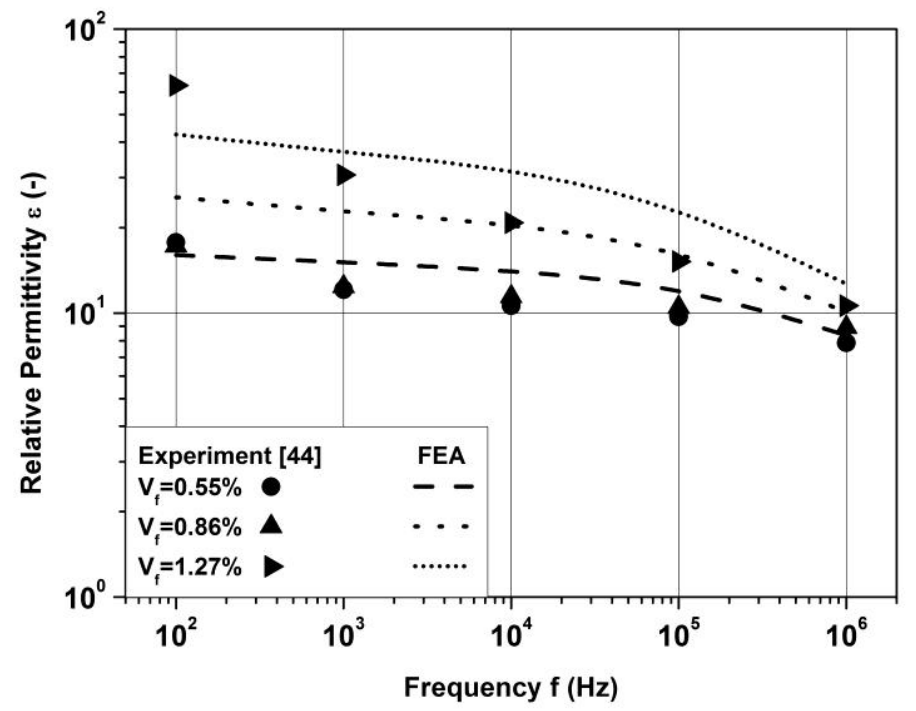

